\title{
POR UNA CONCEPCIÓN MODERNA DE LA ENSEÑANZA DE LA FÍSICA
}

\author{
RAMAL ZEA, JOSÉ M. \\ Agregado de Física y Química. IES Alhadra. Almería.
}

SUMMARY

In this paper it is suggested how to approach, from a modern viewpoint, some questions, which are usually worked in the Physics lessons. Some particular activities are proposed and discussed.

\section{INTRODUCCIÓN}

En la parte dedicada a la física de los textos que conocemos de física y química de $3^{\circ}$ de BUP y de $1^{\circ}$ de bachillerato de la LOGSE, los ejemplos que se utilizan, las situaciones que se consideran son, por decirlo de una manera suave, demasiado antiguos. Se echa en falta en estos textos una concepción moderna de la física en la línea apuntada por Moreira (1990). Esto, como se sabe, constituye un vigoroso material polémico para quienes sostienen que la educación formal está divorciada del mundo «real» (Claxton, 1994).

Al hojear uno cualquiera de esos textos, parece que estuviéramos en el siglo pasado, pues se ignoran sistemáticamente desarrollos y rupturas ocurridos en física, algunos ya muy antiguos (relatividad, teoría cuántica) y otros más recientes (caos). Y esto no se ha solucionado con la introducción de temas dedicados a la Relatividad y a la física cuántica en los programas. Lo que sugerimos es, a la vez, más simple y más complicado. Se trataría, por ejemplo, de trabajar la mecánica clásica, pero planteando, en los momentos convenientes, actividades en que se cuestionen sus supuestos fundamentales. De este modo, en nuestra modesta opinión, los temas clásicos aparecerían bajo una nueva luz, que haría ver sus insuficiencias y sus límites. Incluso sistemas tan clásicos, como el péndulo sin rozamiento, en contextos insólitos, pueden abrir a la imaginación puntos de vista no habituales de una gran riqueza.
Pensamos que nuestro planteamiento puede contribuir a transmitir una imagen menos cerrada, más dinámica de la ciencia; es decir, facilita que los alumnos capten que hay preguntas a hacer y que comiencen a pensar no sólo en la respuesta. Por otra parte, el uso de contenidos que den una visión contemporánea de la ciencia resulta motivador para los alumnos y capaz de despertar su interés (Caamaño, 1988; Marco et al., 1990; Solbes, 1996).

\section{PROPUESTA DE DIDÁCTICA}

Vamos a comentar para el tema del movimiento (mecánica), tal y como lo planteamos en $3^{\circ}$ de BUP, cómo se pueden llevar a la práctica algunas de las ideas anteriores. Mostraremos algunas actividades y las iremos comentando. No hay que olvidar que se trata sólo de ejemplos aislados y no deben, pues, entenderse de forma descontextualizada ni al margen del conjunto de objetivos didácticos. Las actividades que proponemos se inspiran en la perspectiva constructivista (Driver, 1988; Novak, 1982). Tratamos de que las actividades sean pretextos para dialogar, pensar y dudar, pues nos parece de escaso interés que los alumnos «conozcan la fórmula» o «reciten la definición», si éstas no han servido para una reflexión previa. 
En este tema del movimiento (mecánica), aunque se distingue cuidadosamente entre descripción y explicación, se trabajan conjuntamente aspectos cinemáticos y dinámicos. Así, la introducción del concepto de velocidad parte de la pregunta: ¿Por qué es tan importante la velocidad de un sistema? La repuesta que se solicita va por el camino de aclarar que es importante, porque el movimiento es una forma de la energía (la energía cinética), lo cual establece un lazo con un tema ya estudiado. En este contexto, e inmediatamente después de la introducción formal de la velocidad como derivada temporal del vector de posición, se plantea la actividad siguiente:

A.1. ¿Qué harías para calcular:

a) La velocidad instantánea de un coche?

b) La velocidad máxima de un atleta que corre los $100 \mathrm{~m}$ ?

c) La velocidad de la luz?

d) La velocidad de un electrón?

Esta actividad tiene el propósito de obligar a traducir físicamente lo que, en este momento, es sólo una definición matemática. Como se ve, las situaciones planteadas corresponden a tres contextos característicos: el mundo de lo grande, el mundo de lo muy pequeño, invisible, y el mundo de lo muy rápido. Todos los casos requieren técnicas diversas para resolverlos.

Este apartado de introducción de la velocidad termina con esta actividad:

A.2. Imagina un cuerpo que, en condiciones ideales, aumenta progresivamente su velocidad. ¿Qué velocidad máxima alcanzará?

La actividad se presta a un rico debate y comentario, pues muchos alumnos responden que la velocidad máxima vendrá determinada por la energía disponible. Por supuesto, que, en cada clase, siempre hay alumnos que conocen la respuesta aceptada. Después del debate, es el momento de introducir $c$ como velocidad máxima y, si se quiere, se puede trabajar en la interpretación de la conocida gráfica $E_{C^{-}} v$ en que se comparan la situación real con la predicha a partir de la fórmula clásica $E_{C}=m v^{2} / 2$

A.3. Imagina que se lanza una pelota con una cierta rapidez inicial $v_{0}$. Si el rozamiento con el aire es despreciable:

Describe cualitativamente el movimiento. ¿Hay aceleración? ¿Cuántas trayectorias son posibles? ¿Cómo se puede controlar la forma de la trayectoria? Esas condiciones, ¿son independientes?

Considera todas las situaciones posibles.

Lo que se pretende con esta actividad es una introducción significativa al movimiento de los proyectiles. En el comentario se resalta que, aunque el movimiento de todos los proyectiles se ajusta a la misma ley, en la naturaleza hay algo más que leyes. Hay también condi- ciones iniciales. Esta distinción, que es importante, se olvida con demasiada frecuencia. Después del estudio de la manera habitual de los proyectiles se plantea la actividad siguiente:

A.4 ¿Qué información se precisa para determinar el comportamiento concreto de un cuerpo según la mecánica de Newton?

Aquí, utilizando como ejemplos los distintos casos que se han trabajado (m.r.u., m.r.u.a., m.c.u., m.a.s.), se hace ver que, en todos ellos, la información es siempre la misma: la ley y las condiciones iniciales.

Por último, se propone una reflexión de más alcance, bajo su apariencia «inocente».

A.5. Se disparan dos proyectiles desde el mismo punto y con la misma rapidez inicial, pero en direcciones ligeramente diferentes. ¿Qué puedes decir sobre sus trayectorias a medida que transcurre el tiempo?

La respuesta, que se razona con dibujos y a partir de las ecuaciones, es que las trayectorias serían sólo ligeramente diferentes. Pero, en el comentario se insiste en que el punto clave está en las palabras subrayadas, que, en realidad, proponen algo imposible (habría que conocer las condiciones iniciales con una precisión infinita), aunque, en la situación comentada, las consecuencias de un pequeño «desvío» no sean importantes.

A continuación vamos a ver cómo un problema clásico lleva a la aparición de uno de los más extraños objetos de la física actual.

A.6. ¿Qué rapidez inicial tiene que tener un móvil para escapar del campo gravitatorio terrestre?

El propósito de la actividad es que los alumnos utilicen el principio de conservación de la energía en un contexto significativo. La respuesta, $v^{2}=2 G M / R$, que se obtiene sin mayor dificultad, se aprovecha para insistir en que este resultado vale para cualquier cuerpo, no sólo para la Tierra, si bien para ciertos cuerpos la velocidad de escape será menor que para la Tierra y, para otros, es mayor. Se pide, entonces, un cálculo aproximado de la velocidad de escape para la Tierra $\left(M_{T}=6.10^{24} \mathrm{~kg}\right.$; $R_{T} \approx 6000 \mathrm{~km} ; G \approx 7.10^{-11}$ U.I.) y, de inmediato, se plantea la actividad siguiente.

A.7. Vamos a imaginar que la Tierra se comprime hasta que su radio sea: a) de $1000 \mathrm{~km}$, b) de $6 \mathrm{~km}$, c) de $9 \mathrm{~mm}$. ¿Cuál sería la velocidad de escape en cada caso? Comenta el resultado.

La actividad sugiere un importante componente de la actividad científica: el uso de la imaginación para inventar lo que pudiera existir (aunque no se sabe si existe). En c), se obtiene para $v_{0}$ un valor superior a $c$. El resultado se interpreta en el sentido de que, para un cuerpo tal, ni la misma luz podría librarse de su campo de atracción. Un cuerpo así, captaría todo lo que pasara, incluida la luz, lo que lo transformaría en un «agujero negro». Se 
pide, entonces, a los alumnos que, a la vista de lo anterior, escriban una definición de «agujero negro» y se pasa a la lectura de un conocido texto de Asimov (1986) para concluir con una pregunta (actividad 8):

A.8. Puesto que un «agujero negro», por definición, es invisible, ¿cómo se podría detectar su presencia?

Esta cuestión conduce a una idea muy importante, la idea de que hay muchas formas en que la materia puede dar a conocer su presencia, y emitir «luz» es sólo una de ellas. De hecho, la única cosa que toda materia tiene que hacer es ejercer una fuerza gravitatoria. La prueba final de la presencia de materia, por tanto, no es si brilla, sino si atrae o no a otra materia. Aquí, es bastante fácil conectar con la investigación actual: búsqueda de «agujeros negros» y de la materia oscura.

En las clases de este nivel se trabajan siempre movimientos que vienen descritos por «limpias» ecuaciones matemáticas. Y nuestras clases no son una excepción.

Pero, en la actualidad, el azar desempeña un papel central en nuestra comprensión de la naturaleza de las cosas. Por esto, resulta muy conveniente trabajar alguna situación por completo aleatoria como la que se plantea en la actividad que sigue.

A.9. Una partícula se mueve en una recta de la siguiente forma: en cualquier intervalo de tiempo, y sin importar el punto en que se encuentre, se desplaza una determinada distancia. En cada transición, el movimiento puede efectuarse hacia la derecha o hacia la izquierda con una probabilidad de transición 1/2. Inicialmente, la partícula está en el origen. Si se observa la partícula cada 30 segundos (en este tiempo el desplazamiento es de una unidad), ¿dónde estará la partícula después de 10 minutos?

En A.9 las matemáticas que manejan los alumnos no sirven. Pero se puede simular el movimiento de la partícula lanzando una moneda y estableciendo que, si sale «cara», el desplazamiento es hacia la derecha $(+1) \mathrm{y}$, si sale «cruz», hacia la izquierda (-1). En la tabla I se recogen datos obtenidos en una experiencia en clase.

Así se comprueba que el movimiento es impredecible, pues una segunda experiencia dará una secuencia distinta, aunque sí podemos prever su comportamiento global, que corresponde a una difusión. Como se ve, en esta actividad se introduce una técnica muy importante (la simulación), que normalmente no se trabaja.
A continuación, se plantea que, como el movimiento de la partícula se ajusta siempre a la misma ley, describan el movimiento para intervalos de 15 segundos (lógicamente ahora los desplazamientos serán menores). Lo que hay que resaltar es que, globalmente, el aspecto del movimiento es el mismo, y que seguirá siendo el mismo si consideramos intervalos de tiempo cada vez más reducidos; es decir, registramos con mayor frecuencia la posición de la partícula (lo que hacemos así es examinar con «lupa» cada vez partes más pequeñas de la trayectoria). La partícula sigue una trayectoria extremadamente complicada e irregular que es también desigual a cualquier escala de tamaño, trayectoria que, como se sabe, recibe la denominación de fractal (Lavenda, 1985).

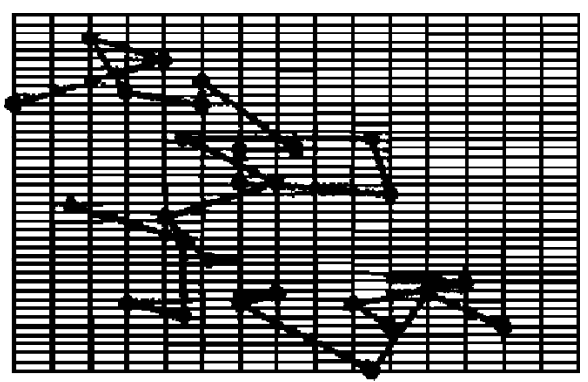

\section{A.10. Estudia el movimiento del gráfico.}

a) ¿Cómo se puede explicar este movimiento?

b) En particular, ¿qué puedes decir sobre la velocidad en el punto núm. 19? El intervalo de tiempo entre dos posiciones sucesivas es de 30 segundos.

Lo que se ha trabajado en A.9 se utiliza ahora para estudiar el movimiento real de una partícula browniana. En lo que se refiere a la explicación del movimiento, a los alumnos se les ocurre que puede ser debido a que la partícula recibe impulsos aleatorios en magnitud y dirección. El propósito de $b$, por otra parte, es hacer ver las limitaciones del concepto de velocidad, pues se trata de usar lo que se sabe en matemáticas sobre derivadas para llegar a la conclusión de que en el punto indicado o en cualquier otro es difícil hablar de una velocidad en el sentido clásico, ya que la trayectoria es en zigzag y no se puede asegurar que el cociente $\Delta \mathrm{s} / \Delta \mathrm{t}$ tienda a un límite fijo cuando consideramos intervalos de tiempo cada vez menores. En suma, el propósito de $b$ es, en cierto modo, inverso del de A.1, pues, si allí lo que se pretendía era dar «cuerpo» a la noción abstracta de derivada, aquí esa noción matemática se usa en toda su complejidad para el estudio de una situación física concreta.

\begin{tabular}{|lrrrrrrrrrrrrrrrrrrrrr} 
Intervalo & $1^{\circ}$ & $2^{\circ}$ & $3^{\circ}$ & $4^{\circ}$ & $5^{\circ}$ & $6^{\circ}$ & $7^{\circ}$ & $8^{\circ}$ & $9^{\circ}$ & $10^{\circ}$ & $11^{\circ}$ & $12^{\circ}$ & $13^{\circ}$ & $14^{\circ}$ & $15^{\circ}$ & $16^{\circ}$ & $17^{\circ}$ & $18^{\circ}$ & $19^{\circ}$ & $20^{\circ}$ \\
Desplazamiento & +1 & +1 & +1 & +1 & -1 & +1 & +1 & +1 & +1 & +1 & -1 & +1 & -1 & +1 & +1 & -1 & -1 & -1 & -1 & +1 \\
& & & & & & & & & & & & & & & & & & & & &
\end{tabular}


Un sistema que también se estudia en las clases de física de este nivel es el péndulo sin rozamiento. Vamos a ver cómo se puede usar para introducir la idea de que un movimiento sometido a una ley completamente determinista se vuelve indeterminado en ciertas condiciones y para recalcar la idea de que el azar forma parte esencial de nuestra descripción de la naturaleza. Consideremos la siguiente actividad:

A.11. Un péndulo consiste en una bola pesada que pende de una varilla rígida de $1 \mathrm{~m}$ de longitud. Este sistema puede oscilar o rotar según las condiciones iniciales. ¿El péndulo, bajo qué condiciones: a) oscila?, b) rota?

En la discusión se establece que el estado del sistema en cualquier instante se puede expresar dando la velocidad y el ángulo $\theta$ con respecto a la posición de equilibrio. El punto esencial es que este movimiento está sometido a una ley: $m g h+m v^{2} / 2=c t e$. Para que rote, su E.C. debe ser lo suficientemente elevada para que no «caiga» antes de alcanzar la vertical: la rotación requiere más energía que la oscilación. Una vez llegados a este punto, se pide a los alumnos la resolución de cuestiones típicas como: Si la velocidad del péndulo, cuando pasa por el punto superior, es de $5 \mathrm{~m} / \mathrm{s}$ en sentido horario, ¿qué velocidad llevará cuando $\theta$ valga $90^{\circ}, 0^{\circ}$, etc.? Y se pasa a la actividad siguiente.

A.12. ¿Qué ocurre si su empuje inicial es exactamente el suficiente para hacerle llegar con velocidad nula a su posición vertical?

En un caso como este, la dirección en que volverá a caer, y así, la naturaleza de su movimiento, es indeterminada: una perturbación infinitesimal basta para arrastrar el sistema, bien hacia una oscilación, bien hacia una rotación. En el comentario se resalta que una trayectoria puede volverse intrínsecamente indeterminada en ciertos puntos singulares. Aquí, resulta muy adecuada la lectura del hermoso texto de Poincaré.

A.13. Una causa muy pequeña, que se nos escapa, determina un efecto considerable que no podemos prever $y$, entonces, decimos que dicho efecto se debe al azar, etc. (Poincaré, 1944)

Por último, vamos a indicar cómo trabajar una situación en que un movimiento regido por una ley determinista se vuelve completamente impredecible e indeterminado.

A.14. Imagina una mesa de billar en cuyo centro hay un obstáculo convexo. Desde un punto cualquiera se lanza una bola que chocará repetidas veces con los bordes y con el obstáculo. Dibujar la trayectoria de la bola. Desde el mismo lugar que antes se lanza una segunda bola con la misma rapidez, pero en una dirección ligeramente diferente. Dibujar la trayectoria de esta segunda bola.

El dibujo muestra que, después de unas pocas reflexiones, las dos trayectorias son completamente distintas. Este sistema, el billar de Sinaï (Eckmann, 1991), que puede parecer muy abstracto y alejado de la realidad, forma, aunque ellos no lo saben, parte de la vida cotidiana de los alumnos, pues, ¿quién no conoce la máquina de bolas? Esta máquina siempre da un juego distinto, sin que importe lo cuidadosamente que un «mago» del mismo intente reproducir el impulso dado a la bola de acero. En el comentario de la actividad se aprovecha esto para destacar que muchos sistemas físicos dependen de forma sensible de las condiciones iniciales, cualesquiera que sean esas condiciones. Dicho de otra forma, un cambio pequeño en las condiciones iniciales conduce a un gran cambio en el comportamiento del sistema a largo plazo. Aquí se hace una comparación con A.5 en donde la conclusión era muy distinta y se subraya que el estado de un sistema no puede conocerse nunca: hay una incertidumbre intrínseca. En ambas actividades las técnicas utilizadas son un dibujo cuidadoso y la discusión heurística para hacer las cosas plausibles, pero sin dar una auténtica demostración. A partir de A.14 es fácil conectar con problemas como el de la predicción del tiempo o el del cambio climático. Se pueden usar textos como «El efecto mariposa» (Lorenz, 1995), recortes de prensa, etc.

\section{REFLEXIONES FINALES}

No sabemos hasta qué punto habremos logrado nuestro propósito de mostrar cómo, adoptando un enfoque actual, se pueden renovar cuestiones bastante manidas.

Terminaremos con una reflexión, pero no sin antes transcribir dos comentarios (que constituyen los dos extremos de un espectro) de una alumna y un alumno, respectivamente, en su respuesta a la encuesta que se les pasa al final de cada tema en que se les pregunta si les ha gustado o no y se les pide que expliquen sus razones:

«Me ha gustado porque en todo momento he tenido que estar con la mente activa y muy despierta.»

«Por lo menos, algunos ejercicios de los que hemos hecho se salen de la rutina de siempre.»

Ahora bien, la investigación didáctica ha puesto de manifiesto la importancia que, para conseguir un aprendizaje significativo, tienen las actitudes de los alumnos, pues una actitud positiva determina una mayor comprensión y unos mejores resultados (Gil et al., 1991). Aunque no hemos evaluado nuestros materiales y es, por tanto, sólo una opinión personal, creemos que los alumnos que trabajan según hemos dicho muestran una mejora en su actitud hacia la física. En efecto, un aprendizaje planteado de forma más abierta y en el que la construcción de conocimientos se presenta un poco como una aventura, es (creemos) de una gran carga motivadora y tiene alguna probabilidad de «enganchar» a los alumnos. El inconveniente de nuestra propuesta puede ser que, a veces, no sepamos medir bien las capacidades o posibilidades de nuestros alumnos, con el consiguiente peligro de rechazo. De todas formas, el proceso de enseñanzaaprendizaje es un asunto complejo y no hay que pensar que con sólo un pequeño cambio como el que sugerimos se resuelvan todos los problemas. 


\section{REFERENCIAS BIBLIOGRÁFICAS}

ASIMOV, I. (1986). Cien preguntas básicas sobre la ciencia. Madrid: Alianza editorial.

CAAMAÑO, A. (1988). Tendencias actuales en el currículo de ciencias. Enseñanza de las ciencias, Vol. 6(3), pp. 265-277.

CLAXTON, G. (1994). Educarmentes curiosas. Madrid: Visor.

DRIVER, R.(1988). Un enfoque constructivista para el desarrollo del currículo en ciencias. Enseñanza de las ciencias, 6(2), pp. 109-120.

ECKMANN, J.P. y MASHAAL, M. (1991). La física del desorden. Mundo científico, 115, pp. 722-730.

GIL, D., CARRASCOSA, J., FURIÓ, C. y MARTÍNEZTORREGROSA, J. (1991). La enseñanza de las ciencias en la educación secundaria. Barcelona: ICE/Horsori.
LAVENDA, R.H.(1985). El movimientobrowniano.Investigación y ciencia, 103, pp. 36-45.

LORENZ, E.N. (1995). La esencia del caos. Madrid: Debate

MARCO, B., MARTÍN-MOLTALVO, J., PARAMIO, M.L. y MACÍAS, R. (1990). La actualidad científica en el diseño curricular de las ciencias experimentales. Madrid: Narcea.

MOREIRA, M. A. (1990). Um mapa conceitual para interações fundamentais. Enseñanza de las ciencias, 8(4), pp. 133-139.

NOVAK, J.D. (1982). Teoría y práctica de la educación. Madrid: Alianza.

POINCARÉ, H. (1944). Ciencia y método. Madrid: Espasa.

SOLBES, J. (1996). La física moderna y su enseñanza. Alambique, 5, pp. 59-67.

[Artículo recibido en julio de 1997 y aceptado en julio de 1998.] 